\title{
Association between tumour infiltrating lymphocytes, histotype and clinical outcome in epithelial ovarian cancer
}

Fiona R. James ${ }^{1,3^{*}}$ (D), Mercedes Jiminez-Linan², Jennifer Alsop ${ }^{3}$, Marie Mack ${ }^{3}$, Honglin Song ${ }^{3}$, James D. Brenton ${ }^{3}$, Paul D. P. Pharoah ${ }^{4}$ and H. Raza Ali ${ }^{5}$

\begin{abstract}
Background: There is evidence that some ovarian tumours evoke an immune response, which can be assessed by tumour infiltrating lymphocytes (TILs). To facilitate adoption of TILs as a clinical biomarker, a standardised method for their H\&E visual evaluation has been validated in breast cancer.

Methods: We sought to investigate the prognostic significance of TILs in a study of 953 invasive epithelial ovarian cancer tumour samples, both primary and metastatic, from 707 patients from the prospective population-based SEARCH study. TILs were analysed using a standardised method based on H\&E staining producing a percentage score for stromal and intratumoral compartments. We used Cox regression to estimate hazard ratios of the association between TILs and survival.

Results: The extent of stromal and intra-tumoral TILs were correlated in the primary tumours $(n=679$, Spearman's rank correlation $=0.60, P<0.001$ ) with a similar correlation in secondary tumours $(n=224$, Spearman's rank correlation $=0.62, P<0.001$ ). There was a weak correlation between stromal TIL levels in primary and secondary tumour samples (Spearman's rank correlation $=0.29, P<0.001$ ) and intra-tumoral TIL levels in primary and secondary tumour samples (Spearman's rank correlation $=0.19, P=0.0094$ ). The extent of stromal TILs differed between histotypes (Pearson chi2 (12d.f.) 54.1, $P<0.0001$ ) with higher levels of stromal infiltration in the high-grade serous and endometriod cases. A significant association was observed for higher intratumoral TIL levels and a favourable prognosis (HR 0.74 95\% Cl 0.55-1.00 p = 0.047).

Conclusion: This study is the largest collection of epithelial ovarian tumour samples evaluated for TILs. We have shown that stromal and intratumoral TIL levels are correlated and that their levels correlate with clinical variables such as tumour histological subtype. We have also shown that increased levels of both intratumoral and stromal TILs are associated with a better prognosis; however, this is only statistically significant for intratumoral TILs. This study suggests that a clinically useful immune prognostic indicator in epithelial ovarian cancer could be developed using this technique.
\end{abstract}

Keywords: Tumour infiltrating lymphocytes (TILs), Epithelial ovarian cancer, Standardised method, Histological subtype, Survival time

\footnotetext{
* Correspondence: fiona.james11@nhs.net

1 Lancashire Teaching Hospitals Foundation NHS Trust, Lancashire, UK

${ }^{3}$ Department of Oncology, University of Cambridge, Cambridge, UK

Full list of author information is available at the end of the article
} 


\section{Background}

Ovarian carcinoma is the fourth most common cancer in women worldwide but it is responsible for the most fatalities due to gynaecological malignancy in the developed world [1]. Known prognostic factors include tumour characteristics (grade, histotype, clinical stage), patient factors (age, fitness, smoking status), treatment factors, and the response to first line treatment [2].

There is evidence that some epithelial ovarian tumours evoke an immune response and that this affects prognosis [3]. Several studies have investigated the effect of various subtypes of immune cell response on prognosis in ovarian cancer [4-20]. These studies have been of modest sample sizes ranging from 9 through to 500 tumours. Immunohistochemistry was used to investigate the immune response, but they have shown conflicting results (outlined in Table 1), though most suggest that CD3(+) and CD8(+) tumour infiltrating lymphocytes are associated with a favourable outcome. Immune infiltration has also been shown to be enriched in some molecular subtypes of ovarian cancer [21]. The anti-tumour immune response is perhaps most easily assessed morphologically using standard histopathology based on haematoxylin and eosin (H\&E) staining. Therefore this may represent the most promising avenue for clinical translation. However, no study has yet investigated the effect of total tumour infiltrating lymphocytes (TILs) on prognosis in ovarian cancer.

There have been calls for the use of a standardised method of evaluating TILs in cancer and incorporating it clinically as a prognostic variable [22]. Haematoxylin and eosin (H\&E) staining and visual analysis is a recognised cheap and clinically accessible method of evaluating TILs. A standardised method for H\&E visual evaluation of TILs has been validated in the context of breast cancer [23].

We investigated the pathological and prognostic significance of TILs in epithelial ovarian cancer. We used a standardised method of TIL evaluation and a large prospective population-based study of epithelial ovarian cancer to achieve this.

\section{Methods}

\section{Study population}

The SEARCH ovarian cancer study is an ongoing, population-based ovarian cancer case-control study, covering the regions served by the East Anglia and West Midlands cancer registries in the UK. All patients diagnosed in East Anglia with invasive epithelial ovarian cancer under the age of 70 years since 1991, and still alive in 1998 when recruitment started are eligible to participate. Details of this study and its participants have been published previously [24]. To date over 2500 women with epithelial ovarian cancer have been recruited. We have retrieved archival, formalin-fixed, paraffin-embedded
(FFPE) tumour tissue from the primary surgery from 707 SEARCH participants with invasive epithelial ovarian cancer. A total of 953 samples have been retrieved including tumour from both primary and metastatic sites.

Survival time data were available through the regional cancer registry which obtains notification of deaths by death certificate flagging through the Office of National Statistics. The lag times for this are a few weeks for cancer deaths and two months to a year for non-cancer deaths. In addition, the cancer registry checks vital status by querying the National Health Service Strategic Tracing Service. Vital status was ascertained at the end of June 2015 and all analyses were censored on 31 December 2014 to allow for delay in reporting of vital status. Ovarian cancer specific mortality was defined as deaths where ovarian cancer was listed as the cause of death on Parts $1 \mathrm{a}, 1 \mathrm{~b}$, or $1 \mathrm{c}$ of the death certificate.

\section{Evaluation of tumour infiltrating lymphocytes}

Formalin-fixed, paraffin-embedded tumour samples were sectioned and stained using hematoxylin and eosin. The morphology was reviewed by a gynaecological pathologist (MJ-L). Full face stained sections were then scanned and the digital images were used for evaluation of TILs.

Evaluation of TILs was performed by manual visual assessment of percentage area covered by lymphocytes, following a standardised method as described in Salgado et al. [23]. In this method the area within the tumour border is selected, the intra-tumoral and stromal compartment are defined, only areas of mononuclear lymphocytic infiltrate are included, and the percentage area is assessed at low ( $4 \times$ objective) and high magnification $(10 \times$ objective). The percentage categories used were $1 \%, 5 \%$ and the nearest $10 \%$ up to $100 \%$. The intra-tumoral and stromal compartments were scored separately. The intra-tumoral and stromal TILs were classified into three groups for the purpose of analysis - very low (1\%), low (5\%) and high $(\geq 10 \%)$. Representative examples of tumours with very low, low and high TIL are shown in Fig. 1.

\section{Statistical analysis}

The proportion of tumours in the three TIL score categories by other histopathologic characteristics was compared using the Pearson $\mathrm{X}^{2}$ - test. Multi-variable Cox regression was used to assess the association between TILs and disease-specific mortality. Follow-up time was adjusted for delayed study entry by using the date of study entry as the start of time at risk (left truncation) [25]. All models were adjusted for FIGO stage (stage 1-4 treated as an ordinal variable), histotype (seven categories) and age at diagnosis. Analyses were censored at 10-years of follow-up or on death from a cause other than ovarian cancer. Stata 14 (Stata Corp, College Station, Texas, USA) statistical software was used for all analyses. 


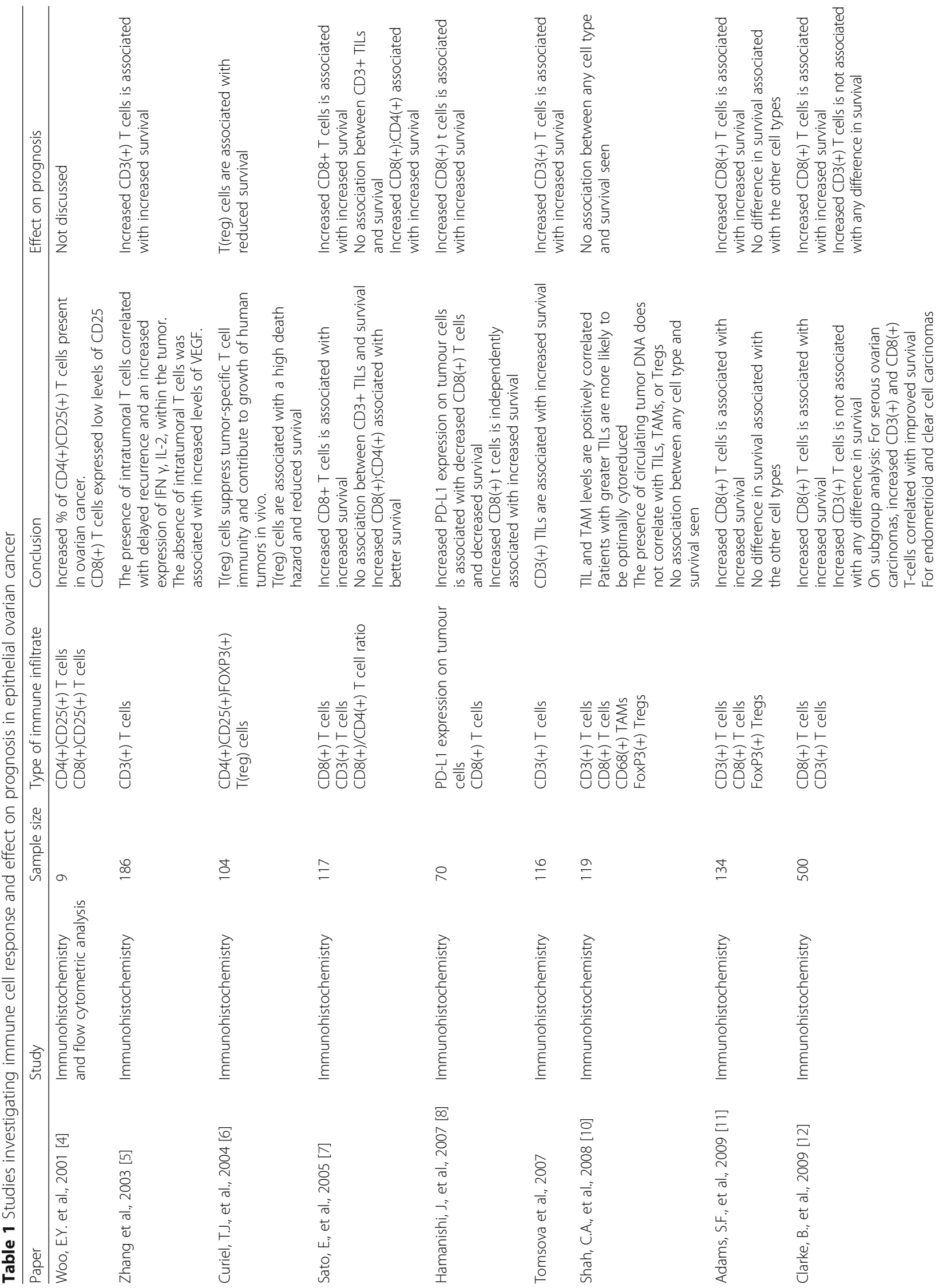



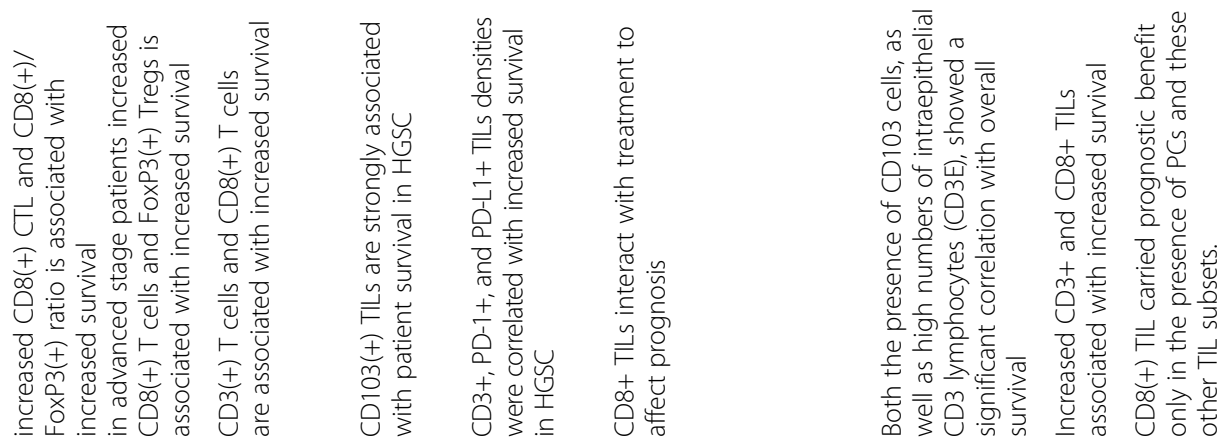

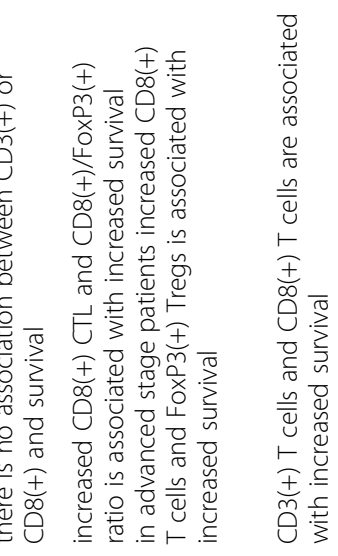
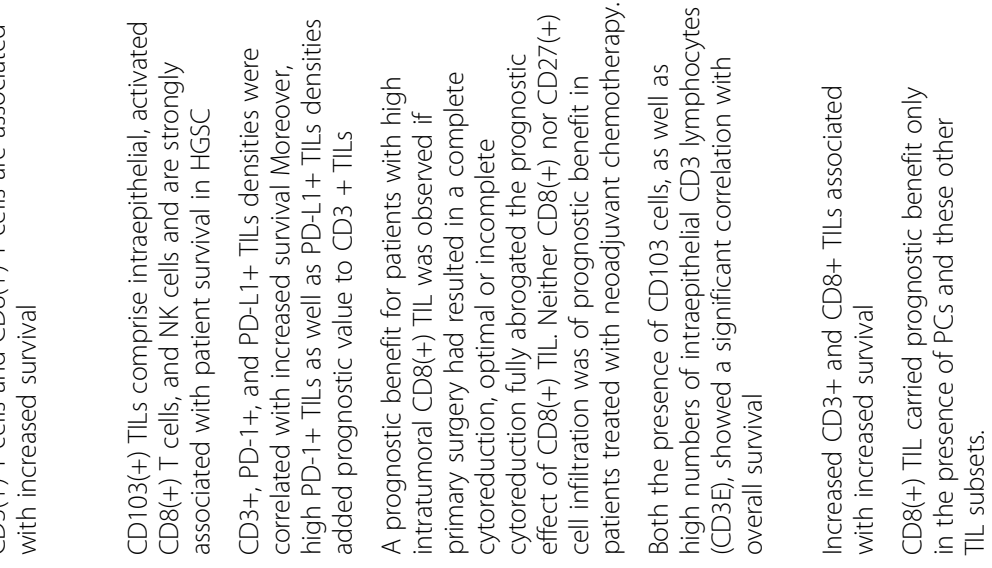

98.

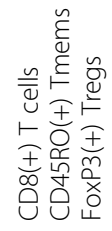

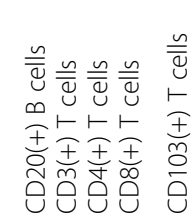

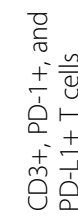

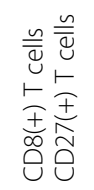

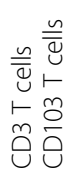

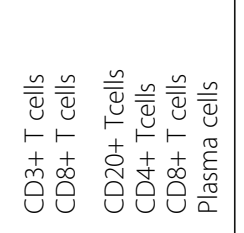

ஓ

$\stackrel{8}{-}$

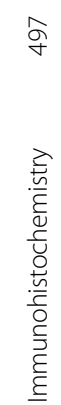

$\stackrel{\llcorner}{\sim}$

$\Sigma$

$\stackrel{n}{m}$

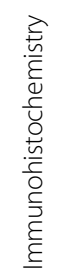

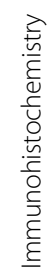

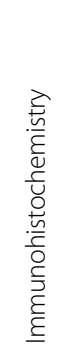

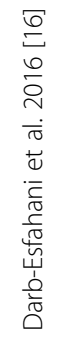

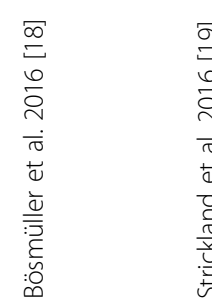

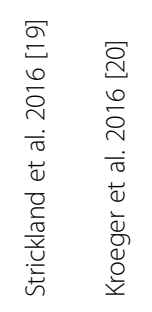

$\stackrel{\llcorner}{\sim}$

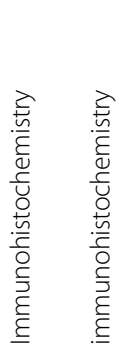

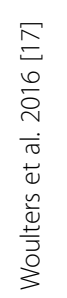




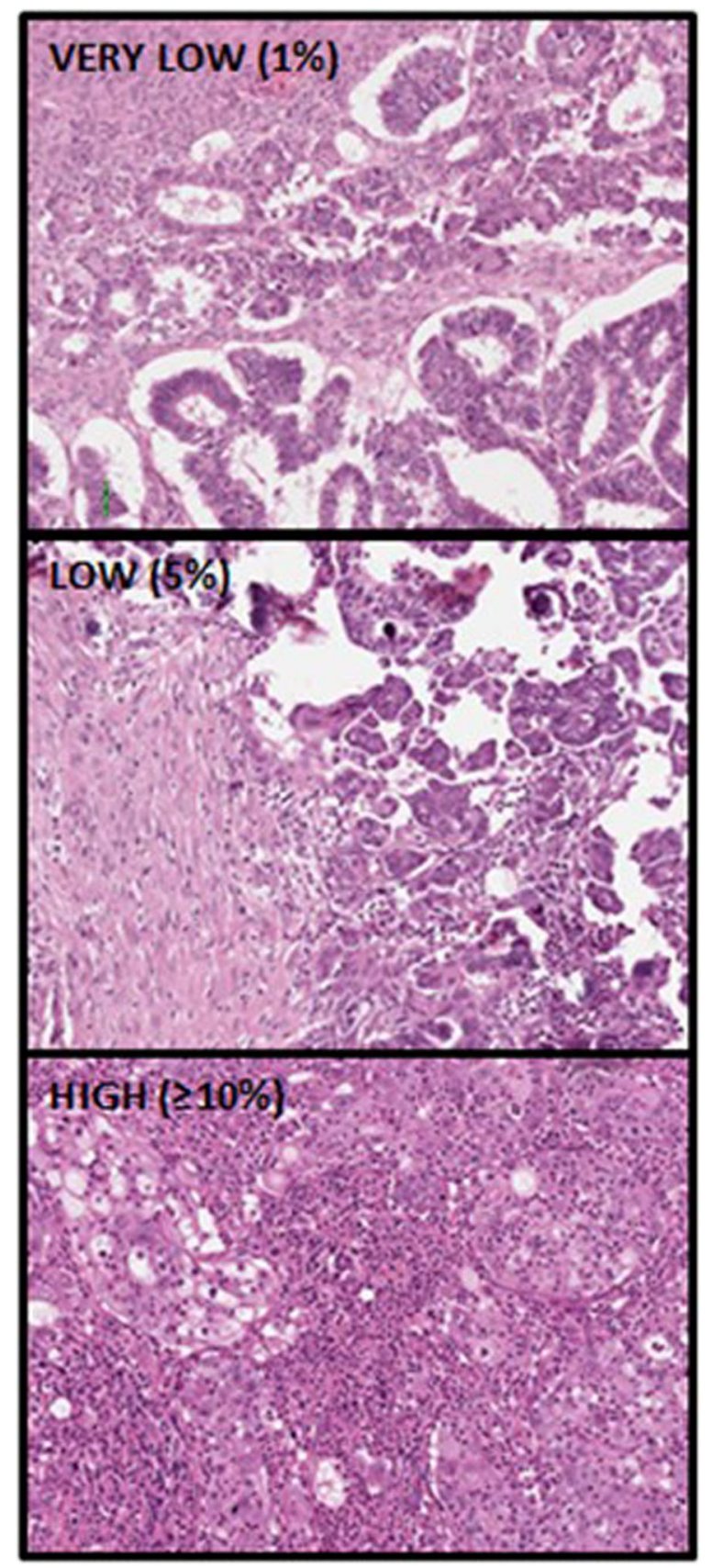

Fig. 1 Representative examples of tumours with very low, low and high TIL

\section{Results}

Data were available for 953 invasive epithelial ovarian tumours (primary and/or metastatic), from 707 women. Of these, primary tumour material was available for 682 cases, omental tumour material for 161 cases and other metastasis material was available for 114 cases (Fig. 2). The clinical characteristics (age at diagnosis and stage) of these cases by histotype are shown in Table 2 .

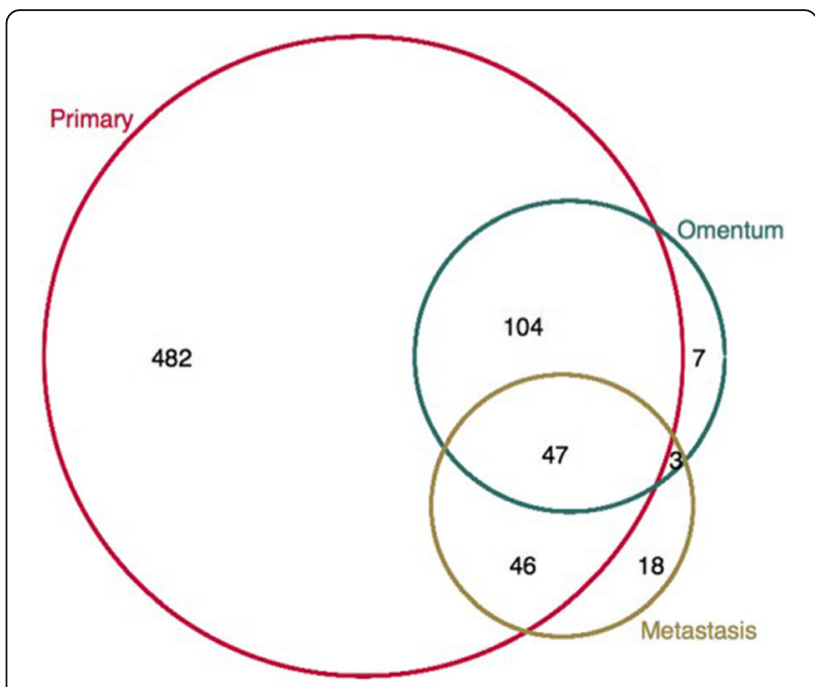

Fig. 2 Number of patients by available tumour sample

There was a moderately strong correlation between the extent of stromal and intra-tumoral TIL levels in the primary tumour $(n=679$, Spearman's rank correlation $=0.60$, $P<0.001)$ with a similar correlation in secondary tumours $(n=224$, Spearman's rank correlation $=0.62, P<0.001)$. Secondary tumour samples were either omental metastases or metastases at other sites. We used the data for the omental metastases in preference to data for other metastases if present; otherwise the data from the other metastases was used. We also evaluated the correlation between TIL levels in primary and metastatic tumours using data from the 196 women for whom both a primary and a secondary tumour sample was available. There was a statistically significant but weak correlation between stromal TIL levels in primary and secondary tumour samples (Spearman's rank correlation $=0.29, P<0.001$ ) and intra-tumoral TIL levels in primary and secondary tumour samples (Spearman's rank correlation $=0.19, P=0.0094)($ Table 3$)$.

Table 2 Clinical characteristics of ovarian cancer cases included in this study

\begin{tabular}{|c|c|c|c|c|c|}
\hline \multirow[t]{2}{*}{ Histotype } & \multirow{2}{*}{$\begin{array}{l}\text { Mean age } \\
\text { at diagnosis }\end{array}$} & \multicolumn{2}{|l|}{ Stage } & \multirow[b]{2}{*}{ Unknown } & \multirow[b]{2}{*}{ Total } \\
\hline & & $|/| \mid\left(\%{ }^{a}\right)$ & III/IV (\%) & & \\
\hline LGSC & 54.0 & $5(25)$ & $15(75)$ & 3 & 23 \\
\hline HGSC & 57.5 & $80(29)$ & $192(71)$ & 42 & 314 \\
\hline Mucinous & 52.7 & $39(91)$ & $4(9)$ & 5 & 48 \\
\hline Endometrioid & 54.5 & $113(91)$ & $11(9)$ & 11 & 135 \\
\hline Clear cell & 55.7 & 70 (91) & $7(9)$ & 5 & 82 \\
\hline Other & 56.3 & $39(64)$ & $22(36)$ & 8 & 69 \\
\hline Borderline & 49.6 & 33 (100) & $0(0)$ & 3 & 36 \\
\hline Total & 55.7 & $379(60)$ & $251(40)$ & 77 & 707 \\
\hline
\end{tabular}

LGSC low-grade serous cancer, HGSC high-grade serous cancer apercent of those with known stage 
Table 3 Association between tumour infiltrating lymphocyte levels in primary and secondary tumours for stromal and intratumoral infiltrating lymphocytes

\begin{tabular}{lllll}
\hline $\begin{array}{l}\text { Primary tumour } \\
\text { infiltrating lymphocytes }\end{array}$ & \multicolumn{4}{l}{ Secondary tumour infiltrating lymphocytes } \\
\cline { 2 - 5 } & $<5 \%$ & $5-9 \%$ & $\geq 10 \%$ & Total \\
\hline Stromal & 21 & 25 & 39 & 85 \\
$<5 \%$ & 7 & 17 & 39 & 63 \\
$5-9 \%$ & 4 & 8 & 37 & 49 \\
$\geq 10 \%$ & 32 & 50 & 115 & 197 \\
Total & & & & \\
Intra-tumoral & 96 & 55 & 19 & 170 \\
$<5 \%$ & 6 & 4 & 5 & 15 \\
$5-9 \%$ & 3 & 5 & 3 & 11 \\
$\geq 10 \%$ & 105 & 64 & 27 & 196 \\
Total & & & & \\
\hline
\end{tabular}

The extent of stromal TIL levels differed between histotypes (Table 4, Pearson chi2 (12d.f.) 54.1, $P<0.0001$ ) with higher levels of stromal infiltration in the high-grade serous and endometriod cases. However, there was little difference in the degree of intra-tumoral infiltrating lymphocytes by histotype (Pearson chi2 (12d.f.) $=15.0, P=0.24$ ).

We assessed the impact of tumour infiltrating lymphocytes on ovarian cancer specific mortality after diagnosis using multi-variable Cox proportional hazards regression.

Table 4 Intra-tumoral and stromal tumour infiltrating lymphocyte levels by histotype

\begin{tabular}{lllll}
\hline Histotype & \multicolumn{4}{l}{ Tumour infiltrating lymphocyte level Number (\%) } \\
\cline { 2 - 5 } & $<5 \%$ & $5-9 \%$ & $\geq 10 \%$ & Total \\
\hline Stromal & & & & \\
Low-grade serous & $13(57)$ & $8(35)$ & $2(9)$ & 23 \\
High-grade serous & $122(39)$ & $85(27)$ & $107(34)$ & 314 \\
Mucinous & $28(58)$ & $12(25)$ & $8(17)$ & 48 \\
Endometrioid & $68(50)$ & $29(22)$ & $38(28)$ & 135 \\
Clear cell & $58(71)$ & $12(15)$ & $12(14)$ & 82 \\
Other & $33(48)$ & $18(26)$ & $18(26)$ & 69 \\
Borderline & $30(83)$ & $4(11)$ & $2(6)$ & 36 \\
Total & $352(50)$ & $168(24)$ & $187(26)$ & 707 \\
Intra-tumoral & & & & \\
Low-grade serous & $20(87)$ & $1(4)$ & $2(9)$ & 23 \\
High-grade serous & $240(76)$ & $47(15)$ & $27(9)$ & 314 \\
Mucinous & $43(90)$ & $3(6)$ & $2(4)$ & 48 \\
Endometrioid & $108(80)$ & $20(15)$ & $7(5)$ & 135 \\
Clear cell & $69(84)$ & $8(10)$ & $5(6)$ & 82 \\
Other & $58(84)$ & $8(12)$ & $3(4)$ & 69 \\
Borderline & $34(94)$ & $2(6)$ & $0(0)$ & 36 \\
Total & $572(81)$ & $89(13)$ & $46(7)$ & 707 \\
\hline
\end{tabular}

The extent of tumour infiltrating lymphocytes in three categories $(<5 \%, 5-9 \%, \geq 10 \%)$ was treated as an ordinal variable in the model. An increase in stromal TIL levels was associated with a slightly better prognosis but the difference was not statistically significant (HR 0.87 95\% CI $0.74-1.02 p=0.091$ ). A slightly stronger and nominally significant association was observed for intra-tumoral TIL levels (hazard ratio $=0.74, P=0.047$ ). When the extent of both stromal and intra-tumoral TIL levels were included in a multi-variable model the effect of the intra-tumoral TIL levels was similar (HR 0.76 95\% CI $0.58-1.00$ $p=0.047$ ), but the association of the stromal lymphocytes was attenuated (HR $0.9795 \%$ CI $0.79-1.19 p=0.78$ ). There was little evidence for heterogeneity of effect of intra-tumoral TIL levels by histotype, although the effect was strongest in the high-grade serous cases (HR 0.70 95\% CI 0.50-0.97 $p=0.034$ ).

\section{Discussion}

This study is the largest collection of epithelial ovarian tumour samples evaluated for TILs, with almost double the number of tumour samples as the largest published study to date [12]. This is also the first time this standardised H\&E visual assessment method of TIL evaluation has been used in ovarian tumours.

We have shown that TILs as assessed by stromal lymphocyte levels correlate with clinical variables such as tumour histological subtype. However, intratumoral TILs did not. We have also shown that increased levels of both intratumoral and stromal TILs are associated with a better prognosis; however, this is only statistically significant for intratumoral TILs.

These results add weight to the argument that the immune system plays an important role in ovarian cancer. They also support the idea that different subtypes of ovarian cancer are variably immunogenic and that the immune response can help define different subtypes.

The difference between stromal and intratumoral TIL effects, despite their correlated levels in both primary and secondary tumours, is interesting, and warrants further investigation. The greater prognostic effect of intratumoral TILs may explained by the biological rationale that T-cell activation necessitates physical contact with target cells in order to engage the T cell receptor [26]. In contrast, the lesser prognostic effect of stromal TILs might be explained in two ways; either they are in-transit to tumour cells hence will eventually become intratumoral TILs, or that they have been 'excluded' from the tumour microenvironment by factors secreted by tumour cells and/or other stromal cells, as a means of evading immune attack [26]. Further work to elucidate the distinct roles of intratumoral and stromal TILs is needed.

The results suggest that a clinically useful immune prognostic indicator in epithelial ovarian cancer could 
be developed using this technique, but larger scale studies are needed to replicate these results. Thus, further studies evaluating the potential of TILs as predictors of treatment response are warranted.

\section{Conclusion}

This study is the largest collection of epithelial ovarian tumour samples evaluated for TILs. We have shown that stromal and intratumoral TIL levels are correlated and that their levels correlate with clinical variables such as tumour histological subtype. We have also shown that increased levels of both intratumoral and stromal TILs are associated with a better prognosis; however, this is only statistically significant for intratumoral TILs. This study suggests that a clinically useful immune prognostic indicator in epithelial ovarian cancer could be developed using this technique.

\section{Abbreviations \\ H\&E: Hematoxylin and eosin; ITL: Intratumoral lymphocytes; SL: Stromal lymphocytes; TILs: Tumour infiltrating lymphocytes}

\section{Acknowledgements}

We thank all the patients who contributed to this study and the SEARCH team for patient recruitment and the staff of the National Cancer Registration Service East for providing access to patient clinical data.

\section{Funding}

This study was funded by Cancer Research UK (C490/A16561).

The funders of this project had no role in the design of the study and collection, analysis, and interpretation of data and in writing the manuscript.

\section{Availability of data and materials}

The data presented in this manuscript are available at. https://doi.org/10.17863/CAM.7140

\section{Authors' contributions}

Project conception and design: HRA, JDB and PDPP. Pathology review: HRA and MJ-L. Processing of pathology material and generation of pathology data: JA, HS and MM. Management of the SEARCH clinical database: HS. Data analysis: FRJ and PDPP. Drafting of the manuscript: FRJ, HRA and PDPP. All authors reviewed and approved the final version of the manuscript

\section{Ethics approval and consent to participate}

SEARCH is approved by the Cambridgeshire 4 Research Ethics

Committee. All participants provided written informed consent.

\section{Consent for publication}

Not applicable.

\section{Competing interests}

The authors declare that they have no competing interests.

\section{Publisher's Note}

Springer Nature remains neutral with regard to jurisdictional claims in published maps and institutional affiliations.

\footnotetext{
Author details

${ }^{1}$ Lancashire Teaching Hospitals Foundation NHS Trust, Lancashire, UK. 2Department of Pathology, University of Cambridge, Cambridge, UK. ${ }^{3}$ Department of Oncology, University of Cambridge, Cambridge, UK ${ }^{4}$ Department of Oncology, Department of Public Health and Primary Care, University of Cambridge, Cambridge, UK. ${ }^{5}$ Department of Pathology, CRUK Cambridge Institute, University of Cambridge, Cambridge, UK.
}

Received: 5 March 2017 Accepted: 22 August 2017

Published online: 20 September 2017

\section{References}

1. Jemal A, et al. Global cancer statistics. CA Cancer J Clin. 2011;61(2):69-90.

2. Agarwal R, Kaye SB. Prognostic factors in ovarian cancer: how close are we to a complete picture? Ann Oncol. 2005;16(1):4-6.

3. Kandalaft $L E$, et al. Immunotherapy for ovarian cancer: what's next? J Clin Oncol. 2011;29(7):925-33.

4. Woo EY, et al. Regulatory CD4(+)CD25(+) T cells in tumors from patients with early-stage non-small cell lung cancer and late-stage ovarian cancer. Cancer Res. 2001;61(12):4766-72.

5. Zhang L, et al. Intratumoral T cells, recurrence, and survival in epithelial ovarian cancer. N Engl J Med. 2003;348(3):203-13.

6. Curiel $\mathrm{TJ}$, et al. Specific recruitment of regulatory $T$ cells in ovarian carcinoma fosters immune privilege and predicts reduced survival. Nat Med. 2004;10(9):942-9.

7. Sato $E$, et al. Intraepithelial CD8+ tumor-infiltrating lymphocytes and a high $\mathrm{CD} 8+/$ regulatory T cell ratio are associated with favorable prognosis in ovarian cancer. Proc Natl Acad Sci U S A. 2005;102(51):18538-43.

8. Hamanishi J, et al. Programmed cell death 1 ligand 1 and tumor-infiltrating CD8+ T lymphocytes are prognostic factors of human ovarian cancer. Proc Natl Acad Sci U S A. 2007;104(9):3360-5.

9. Tomsova $\mathrm{M}$, et al. Prognostic significance of CD3+ tumor-infiltrating lymphocytes in ovarian carcinoma. Gynecol Oncol. 2008;108(2):415-20.

10. Shah CA, et al. Intratumoral T cells, tumor-associated macrophages, and regulatory T cells: association with p53 mutations, circulating tumor DNA and survival in women with ovarian cancer. Gynecol Oncol. 2008:109(2):215-9.

11. Adams SF, et al. Intraepithelial T cells and tumor proliferation: impact on the benefit from surgical cytoreduction in advanced serous ovarian cancer. Cancer. 2009;115(13):2891-902.

12. Clarke B, et al. Intraepithelial T cells and prognosis in ovarian carcinoma: novel associations with stage, tumor type, and BRCA1 loss. Mod Pathol. 2009;22(3):393-402.

13. Leffers $\mathrm{N}$, et al. Prognostic significance of tumor-infiltrating T-lymphocytes in primary and metastatic lesions of advanced stage ovarian cancer. Cancer Immunol Immunother. 2009:58(3):449-59.

14. Stumpf M, et al. Intraepithelial CD8-positive T lymphocytes predict survival for patients with serous stage III ovarian carcinomas: relevance of clonal selection of T lymphocytes. Br J Cancer. 2009;101(9):1513-21.

15. Webb JR, et al. PD-L1 expression is associated with tumor-infiltrating $T$ cells and favorable prognosis in high-grade serous ovarian cancer. Gynecol Oncol. 2016 May:141 (2):293-302.

16. Darb-Esfahani S, et al. Prognostic impact of programmed cell death-1 (PD-1) and PD-ligand 1 (PD-L1) expression in cancer cells and tumor-infiltrating lymphocytes in ovarian high grade serous carcinoma. Oncotarget. 2016 Jan 12;7(2):1486-99.

17. Woulters MC, et al. Size matters: survival benefit conferred by intratumoral $T$ cells is dependent on surgical outcome, treatment sequence and T cell differentiation. Oncoimmunology. 2016 Jan 4;5(5):e1122863.

18. Bösmüller $\mathrm{HC}$, et al. Combined Immunoscore of CD103 and CD3 identifies long-term survivors in high-grade serous ovarian cancer. Int J Gynecol Cancer. 2016 May:26(4):671-9.

19. Strickland KC, et al. Association and prognostic significance of BRCA1/2mutation status with neoantigen load, number of tumor-infiltrating lymphocytes and expression of PD-1/PD-L1 in high grade serous ovarian cancer. Oncotarget. 2016 Mar 22;7(12):13587-98.

20. Kroeger DR, et al. Tumor-infiltrating plasma cells are associated with tertiary lymphoid structures, Cytolytic T-cell responses, and superior prognosis in ovarian cancer. Clin Cancer Res. 2016 Jun 15;22(12):3005-15.

21. Tothill RW, et al. Novel molecular subtypes of serous and endometrioid ovarian cancer linked to clinical outcome. Clin Cancer Res. 2008;14(16):5198-208.

22. Galon J, et al. Towards the introduction of the 'Immunoscore' in the classification of malignant tumours. J Pathol. 2014:232(2):199-209.

23. Salgado $R$, et al. The evaluation of tumor-infiltrating lymphocytes (TILs) in breast cancer: recommendations by an international TILs working group 2014. Ann Oncol. 2015;26(2):259-71

24. Song $\mathrm{H}$, et al. Common variants in mismatch repair genes and risk of invasive ovarian cancer. Carcinogenesis. 2006;27(11):2235-42.

25. Azzato EM, et al. Prevalent cases in observational studies of cancer survival: do they bias hazard ratio estimates? Br J Cancer. 2009;100(11):1806-11.

26. Joyce JA, et al. T cell exclusion, immune privilege, and the tumor microenvironment. Science. 2015. Apr 3;348(6230):74-80. 